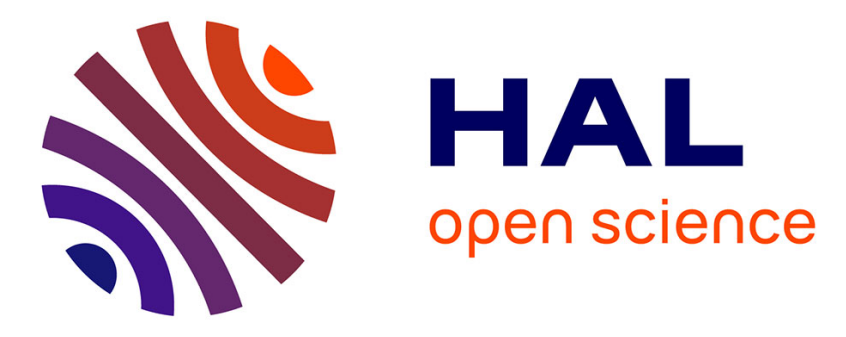

\title{
Spin-orbit coupling as a probe to decipher halogen bonding
}

\author{
Jérôme Graton, Seyfeddine Rahali, Jean-Yves Le Questel, Gilles F Montavon, \\ Julien Pilmé, Nicolas Galland
}

\section{- To cite this version:}

Jérôme Graton, Seyfeddine Rahali, Jean-Yves Le Questel, Gilles F Montavon, Julien Pilmé, et al.. Spin-orbit coupling as a probe to decipher halogen bonding. Physical Chemistry Chemical Physics, 2018, 20 (47), pp.29616-29624. 10.1039/C8CP05690K . hal-02004385

\section{HAL Id: hal-02004385 \\ https://hal.science/hal-02004385}

Submitted on 22 Feb 2022

HAL is a multi-disciplinary open access archive for the deposit and dissemination of scientific research documents, whether they are published or not. The documents may come from teaching and research institutions in France or abroad, or from public or private research centers.
L'archive ouverte pluridisciplinaire HAL, est destinée au dépôt et à la diffusion de documents scientifiques de niveau recherche, publiés ou non, émanant des établissements d'enseignement et de recherche français ou étrangers, des laboratoires publics ou privés. 


\section{Spin-orbit coupling as a probe to decipher halogen bondingt}

Received 00th January 20xx Accepted 00th January $20 x x$ DOI: $10.1039 / \times 0 \times x 00000 x$

www.rsc.org/

\author{
Jérôme Graton, ${ }^{a}$ Seyfeddine Rahali, ${ }^{a}$ Jean-Yves Le Questel, ${ }^{a}$ Gilles Montavon, ${ }^{b}$ Julien Pilmé, ${ }^{* c}$ and \\ Nicolas Galland*a
}

The nature of halogen-bond interactions is scrutinized from the perspective of astatine, the heaviest halogen element. Potentially the strongest halogen-bond donor, its ability is shown to be deeply affected by relativistic effects and especially by the spin-orbit coupling. Complexes between a series of $X Y$ dihalogens $(X, Y=A t, I, B r, C l$ and $F$ ) and ammonia are studied with two-component relativistic quantum calculations, revealing that the spin-orbit interaction leads to a weaker halogenbond donating ability of the diastatine species with respect to diiodine. In addition, the donating ability of the lighter halogen elements, iodine and bromine, in the Atl and AtBr species is more decreased by the spin-orbit coupling than that of astatine. This can only be rationalized from the evolution of a charge-transfer descriptor, the local electrophilicity $\omega_{S, \text { max }}^{+}$, determined for the pre-reactive XY species. Finally, the investigation of the spin-orbit coupling effects by means of quantum chemical topology methods allows us to unveil the connection between the astatine propensity to form charge-shift bonds and the astatine ability to engage in halogen bonds.

\section{Introduction}

Halogen bonds have received considerable interest in recent years due to their unique features and their recognized role in a wide variety of fields from biomedicinal chemistry to materials science. ${ }^{1-5}$ For instance, halogen bonding has been revealed as an important tool in the chemist arsenal to control the synthesis and design of coordination compounds, ${ }^{6}$ as well as one of the "key" weak non-covalent interactions chemists can rely on for structure-based ligand design in medicinal chemistry and agrochemistry. ${ }^{7}$ The halogen bond (XB) is a highly directional interaction, denoted $\mathrm{R}-\mathrm{X} \cdot \mathrm{B}$, between an electron deficient region of the $X$ halogen atom, and an electron rich site of another molecule B, typically a Lewis base. XBs are complex attractive interactions for which charge-transfer as well as electrostatic, dispersion, exchange, etc., components may be claimed. Numerous theoretical investigations have attempted to unveil the nature of this non-covalent interaction, or its leading component(s), ${ }^{8-16}$ but this topic remains the subject of literature controversy. ${ }^{3,17-19}$ Most of these works investigated essentially XBs mediated by light halogen elements. It is well established that the $X B$ strength increases in the order of $\mathrm{F}<\mathrm{Cl}<\mathrm{Br}<\mathrm{l}$. We propose to tackle this topic from the

a. Université de Nantes, CEISAM, UMR CNRS 6230, 2 Rue de la Houssinière, BP 92208, 44322 Nantes Cedex 3, France.E-mail: nicolas.galland@univ-nantes.fr b. IMT Atlantique/Université de Nantes, SUBATECH, UMR CNRS 6457, 4 rue A. Kastler, BP 20722, 44307 Nantes Cedex 3, France.

Sorbonne Université, Laboratoire de Chimie Théorique, F-75005 Paris, France. Email: pilme@lct.jussieu.fr

† Electronic Supplementary Information (ESI) available: three tables reporting (i) supplementary XB interaction energies, (ii) QTAIM descriptors for the XY species, and (iii) calculated spectroscopic constants of the $X Y$ dihalogens. An additional figure displaying correlations between the interaction energies and the $\omega_{s, \max }^{+}$and $V_{\mathrm{s}, \max }$ values calculated at the XB donor $\sigma$-hole. See DOI: 10.1039/x0xx00000x perspective of the heaviest halogen element, astatine (no halogen-specific chemical properties being reported so far for the recently discovered tennessine element ${ }^{20}$ ). It is particularly challenging to investigate experimentally astatine chemistry since it is the rarest element naturally occurring on Earth. ${ }^{21}$ The main advances stem from the interest of ${ }^{211} \mathrm{At}$, the second longest lived isotope, for applications in nuclear medicine. 22 However, it was very recently confirmed through experimental evidences that astatine exhibits a stronger XB donating ability than iodine in the Atl compound. ${ }^{23}$

Astatine is a heavy element $(Z=85)$, and as such it is sensitive to relativistic effects. The relativistic effects are traditionally split in two types of terms, the "scalar" and "spindependent" ones. The scalar-relativistic effects, related to the relativistic mass increase of the inner-core electrons, result in contraction and energetic stabilization of atomic $s$ and $p$ valence shells. The main spin-dependent effect, arising from the interaction of the electron spin with magnetic fields generated by other charged particles in relative motion and leading to the coupling between electron spin and orbital momentum, is the spin-orbit coupling (SOC). It can be of similar magnitude than the scalar-relativistic effects for heavy p-elements such as astatine. Furthermore, the SOC can be as important as the electron correlation and can largely affect the chemical properties of At-containing compounds. ${ }^{24-32}$ For instance, it was predicted that SOC (i) decreases astatine electronegativity by $\sim 10 \%,{ }^{33}$ (ii) favours in solution the affinity of the $\mathrm{AtO}^{+}$species for the nitrogen of thiocyanate ligand instead of the sulfur, ${ }^{34}$ (iii) reverses the bond polarization (i.e. the dipole moment) in the HAt compound, ${ }^{35}$ (iv) weakens by $\sim 40 \%$ the vibrational frequency in $A t_{2},{ }^{36}$ and (v) increases the astatide (At) polarizability by $\sim 20 \% .{ }^{37}$ In the field of study covered here, halogen bonding, computational investigations must include $a$ 
priori both spin-independent and spin-dependent relativistic effects: the interaction energy of At-mediated XBs can be modified by 20 to $35 \%$ upon including the SOC effects. 38,39 Hence, there is an obvious interest in finely analysing the SOC effects on the characteristics of XBs formed by At-compounds, and in trying to unveil the role of SOC on the underlying mechanisms that sustain halogen bonding. As a test set of compounds, we selected in this work complexes between a series of $\mathrm{XY}$ dihalogens $(\mathrm{X}, \mathrm{Y}=\mathrm{At}, \mathrm{I}, \mathrm{Br}, \mathrm{Cl}$ and $\mathrm{F}$ ) and ammonia $\left(\mathrm{NH}_{3}\right)$ for which benchmark quality results have been recently reported. 40

\section{Computational methods}

While scalar-relativistic (sr) calculations can make use of the whole unchanged machinery of non-relativistic quantum chemistry, notably within the effective-core potential (ECP) approaches, the treatment of the spin-orbit interaction requires to expand the wave function with spinors, which are complex vector functions of two or four components. ${ }^{41-43}$ The twocomponent (2c) relativistic density functional theory (DFT) approach was proved reliable for studying many astatinated compounds. ${ }^{33,34,36,44-47}$ Among the available DFT functionals, we have selected the hybrid exchange-correlation PW6B95 functional since (i) it was developed to be accurate for thermochemistry and non-covalent interactions (including $\mathrm{XBs}),{ }^{48}$ and (ii) it was recommended in a recent benchmark study focused on At-species. ${ }^{33}$ The spin-orbit DFT (SODFT) method implemented in the NWChem program package ${ }^{49}$ takes advantage of two-component ECPs, and the inclusion of a spindependent potential into the variational treatment of the oneelectron operator ensures that scalar-relativistic and SOC effects are treated on an equal footing. The small-core energyconsistent ECPnMDF ECPs were used to replace the $n=10,28$ and 60 inner-core electrons of the bromine, iodine and astatine atoms, respectively. ${ }^{50,51}$ Their remaining 25 electrons were dealt with the triple-zeta quality aug-cc-pVTZ-PP basis set, ${ }^{50,51}$ supplemented for the I and At atoms by the $2 \mathrm{c}$ extensions described in ref. ${ }^{52}$. The correlation consistent aug-cc-pVTZ basis set, ${ }^{53-55}$ abbreviated as AVTZ in the following, was used for the lighter atoms, namely $\mathrm{H}, \mathrm{N}, \mathrm{F}$ and $\mathrm{Cl}$.

The energies of the $X Y \cdots N_{3}(X, Y=A t, I, B r, C l$ and $F)$ complexes were corrected from the basis set superposition error (BSSE) using the counterpoise method. ${ }^{56}$ For each $X Y$ dihalogens, the local descriptors of the $\sigma$-hole properties were computed using the TURBOMOLE program package. ${ }^{57} \mathrm{We}$ notably used the finite difference method for calculating the local electrophilicity values. ${ }^{58}$ More precisely, the Fukui function for nucleophilic attack, $f^{+}(r)$, is obtained as:

$f^{+}(r) \approx \rho_{\mathrm{N}+1}(r)-\rho_{\mathrm{N}}(r)$

where $\rho_{\mathrm{N}}$ and $\rho_{\mathrm{N}+1}$ are the electron densities of the neutral species ( $N$ electrons) and of the corresponding anion $(N+1$ electrons) at the geometry of the neutral species. Furthermore, the electronegativity, $\chi=1 / 2(I \mathrm{P}+\mathrm{EA})$, and the hardness,
$\eta=(\mathrm{IP}-\mathrm{EA})$, were obtained from the vertical ionization potential (IP) and electron affinity (EA) values.

Introduced in 1990 by Becke and Edgecombe, ${ }^{59}$ the Electron Localization Function (ELF) is a signature of the distribution of electronic pairs and the analysis of its topology is a powerful tool for the characterization of bonding schemes. ${ }^{60}$ Moreover, the topological analysis of the total electron density, earlier defined by Bader as the Quantum Theory of Atoms-InMolecules (QTAIM), ${ }^{61}$ also provides a route to analyse, evaluate and classify the nature of chemical bonds and interactions. These approaches enable the partition of the 3D molecular space into volumes (or basins) associated to clear physical interpretations. Within the QTAIM approach, only atomic basins of the electron density are observed; a topological atom is defined as the union of a nucleus and of its atomic basin. The atomic charge is readily obtained as the difference between the atomic number (decreased by the charge of the inner-core if ECPs are used) and the electron population of the atomic basin. In contrast, the ELF partition associates electron density with core basins, $C(A)$, around nuclei $A$ and valence basins. These latter are divided into non-bonding basins, $V(A)$, usually corresponding to lone-pairs, and bonding basins, $V\left(A_{1}, A_{2}\right)$, characterizing the covalent character of the bond between two atoms $A_{1}$ and $A_{2}$. The spatial distribution of the valence basins closely matches the non-bonding and bonding domains of the valence shell electron pair repulsion (VSEPR) theory. ${ }^{62}$ Details on the extension of the ELF and QTAIM topological analyses in the framework of 2c-DFT calculations can be found in refs. 29 and 36. All the topological analyses were carried out using modified versions of the NWChem and TopMod09 program packages. ${ }^{49,63}$

In order to evaluate SOC effects on the studied properties, all calculations (geometry optimizations, frequency calculations, topological analyses, etc.) have also been carried out at the sr-DFT level of theory, i.e. in the absence of spindependent potentials in ECPnMDF ECPs.

\section{Results and discussion}

\subsection{When astatine does not follow trends}

Most of the $\mathrm{XY} \cdots \mathrm{NH}_{3}(\mathrm{X}, \mathrm{Y}=\mathrm{At}, \mathrm{I}, \mathrm{Br}, \mathrm{Cl}$ and $\mathrm{F}$ ) complexes have been previously investigated by $\mathrm{Hill}$ and $\mathrm{Hu}, 40$ who provided benchmark quality geometries and interaction energies from counterpoise corrected (CP) CCSD(T)-F12b/VTZ-F12 calculations. However, they assumed that the least electronegative halogen was the only XB donor in the investigated heteronuclear dihalogen systems. Here, we have considered both the $X$ and $Y$ atoms as potential $X B$ donors, limiting ourselves to systems involving at least one At atom (besides $\mathrm{I}_{2}$ and $\mathrm{Br}_{2}$ studied for comparison purposes). All the studied $\mathrm{XY} \cdots \mathrm{NH}_{3}$ complexes exhibit a $\mathrm{C}_{3 \mathrm{v}}$ symmetry (i.e. the halogen and nitrogen atoms are perfectly aligned), with computed intermolecular bond lengths smaller than the sum of the van der Waals radii of the involved atoms (we assumed a $2.02 \AA$ radius for astatine according sr-calculations ${ }^{64}$ ). These are expected features for XB interactions. Note that (i) if a weakly 
Table 1 sr-PW6B95/AVTZ optimized intermolecular and intrahalogen bond lengths, change in the intrahalogen bond length on formation of $\mathrm{XY} \cdots \mathrm{NH}_{3}$, and counterpoise corrected interaction energy (when available, reference CP-CCSD(T)F12b/VTZ-F12 values are given in parentheses ${ }^{a}$ )

\begin{tabular}{lcccc}
\hline & $\mathrm{d}_{\mathrm{\cdots} \cdots \mathrm{N}}(\AA)$ & $\mathrm{d}_{\mathrm{x}-\mathrm{Y}}(\AA)$ & $\Delta \mathrm{d}_{\mathrm{X}-\mathrm{Y}}(\AA)$ & $\Delta E^{\mathrm{CP}}(\mathrm{kcal} / \mathrm{mol})$ \\
\hline $\mathrm{At}-\mathrm{At} \cdots \mathrm{NH}_{3}$ & 2.754 & 2.899 & +0.055 & -9.68 \\
& $(2.782)$ & $(2.900)$ & $(+0.042)$ & $(-9.54)^{b}$ \\
$\mathrm{I}-\mathrm{At} \cdots \mathrm{NH}_{3}$ & 2.717 & 2.820 & +0.061 & -11.04 \\
& $(2.741)$ & $(2.818)$ & $(+0.048)$ & $(-10.90)^{b}$ \\
$\mathrm{Br}-\mathrm{At} \cdots \mathrm{NH}_{3}$ & 2.653 & 2.632 & +0.067 & -14.06 \\
& $(2.666)$ & $(2.625)$ & $(+0.055)$ & $(-13.60)^{b}$ \\
$\mathrm{Cl}-\mathrm{At} \cdots \mathrm{NH}_{3}$ & 2.615 & 2.491 & +0.071 & -15.67 \\
& $(2.630)$ & $(2.479)$ & $(+0.059)$ & $(-15.36)^{b}$ \\
$\mathrm{~F}-\mathrm{At} \cdots \mathrm{NH}_{3}$ & 2.552 & 2.067 & +0.056 & -19.96 \\
& $(2.559)$ & $(2.057)$ & $(+0.047)$ & $(-19.71)^{b}$ \\
$\mathrm{I}-\mathrm{I} \cdots \mathrm{NH}_{3}$ & 2.721 & 2.725 & +0.056 & -8.22 \\
& $(2.767)$ & $(2.720)$ & $(+0.041)$ & $(-7.90)^{b}$ \\
$\mathrm{At}-\mathrm{I} \cdots \mathrm{NH}_{3}$ & 2.770 & 2.807 & +0.049 & -6.99 \\
$\mathrm{Br}-\mathrm{Br} \cdots \mathrm{NH}_{3}$ & 2.542 & 2.350 & +0.062 & -6.98 \\
& $(2.603)$ & $(2.334)$ & $(+0.042)$ & $(-7.40)^{b}$ \\
$\mathrm{At}-\mathrm{Br} \cdots \mathrm{NH}_{3}$ & 2.736 & 2.601 & +0.036 & -4.33 \\
$\mathrm{At}-\mathrm{Cl} \cdots \mathrm{NH}_{3}$ & 2.931 & 2.434 & +0.014 & -1.41
\end{tabular}

a Scalar-relativistic effects were taken into account via ECPs. ${ }^{b}$ Extrapolated to the complete basis set limit, see ref. 40 .

bound $\mathrm{AtCl} \cdots \mathrm{NH}_{3}$ complex can be located at the scalarrelativistic level, it vanishes when the $\mathrm{SOC}$ is taken into account, and (ii) no stable F-mediated XBs with ammonia can be found for the studied complexes. The results of our spin-orbit free calculations on the $\mathrm{XY} \cdots \mathrm{NH}_{3}$ complexes are gathered in Table 1 with the previous scalar-relativistic CP-CCSD(T)-F12b/VTZ-F12 results. ${ }^{40}$ The sr-PW6B95/AVTZ calculations lead to an excellent agreement with the reference values of Hill and $\mathrm{Hu}^{40}$ The intermolecular bond lengths are computed slightly shorter, by $\sim 1.0 \%$, and the average deviation on the interaction energies amounts to $1.1 \%$. Hence, the combination of the PW6B95 functional with AVTZ basis sets appears well suited to the characterization of $\mathrm{XB}$ complexes. The scalar-relativistic calculations corroborate well-accepted trends. For instance, with the increase of the electronegativity difference between the halogen atoms, a monotonous strengthening of the interaction energy and shortening of the intermolecular distance is observed for the five complexes stabilized by an Atmediated XB (rows 1-5, Table 1). Furthermore, within the series of homonuclear dihalogen complexes (rows 1, 6 and 8, Table 1), the decrease in polarizability of the halogen element leads to a decrease of the XB strength. However, we previously pointed out that such trends should be taken with care for astatinated systems. ${ }^{38}$ The spin-dependent relativistic effects can indeed modulate the astatine chemistry out of the territories established by the lighter halogen elements.

The results of our calculations taking into account the relativistic spin-orbit interaction on the previous systems are reported in Table 2. The spin-orbit coupling effect $(\Delta S O)$ is defined as the difference between the 2c-PW6B95/AVTZ and srPW6B95/AVTZ results. At first, one can notice that the interaction energies with ammonia are weakened and, accordingly, the interaction distances are lengthened with the
Table 2 2c-PW6B95/AVTZ optimized intermolecular and intrahalogen bond lengths, change in the intrahalogen bond length on formation of $\mathrm{XY} \cdots \mathrm{NH}_{3}$, and counterpoise corrected interaction energy

\begin{tabular}{ccccc} 
& $\mathrm{d}_{\mathrm{Y} \cdots \mathrm{N}}(\AA)$ & $\mathrm{d}_{\mathrm{X}-\mathrm{Y}}(\AA \AA)$ & $\Delta \mathrm{d}_{\mathrm{X}-\mathrm{Y}}(\AA)$ & $\Delta E^{\mathrm{CP}}(\mathrm{kcal} / \mathrm{mol})$ \\
\hline $\mathrm{At}-\mathrm{At} \cdots \mathrm{NH}_{3}$ & 2.849 & 3.017 & +0.022 & -7.53 \\
$\Delta \mathrm{SO}^{a}$ & +0.095 & +0.118 & -0.033 & +2.15 \\
$\mathrm{I}-\mathrm{At} \cdots \mathrm{NH}_{3}$ & 2.771 & 2.880 & +0.044 & -10.38 \\
$\Delta \mathrm{SO}^{a}$ & +0.053 & +0.061 & -0.017 & +0.66 \\
$\mathrm{Br}-\mathrm{At} \cdots \mathrm{NH}_{3}$ & 2.722 & 2.681 & +0.046 & -12.13 \\
$\Delta \mathrm{SO}^{a}$ & +0.068 & +0.049 & -0.021 & +1.92 \\
$\mathrm{Cl}-\mathrm{At} \cdots \mathrm{NH}_{3}$ & 2.693 & 2.536 & +0.045 & -13.30 \\
$\Delta \mathrm{SO}^{a}$ & +0.078 & +0.045 & -0.026 & +2.37 \\
$\mathrm{~F}-\mathrm{At} \cdots \mathrm{NH}_{3}$ & 2.637 & 2.099 & +0.032 & -15.95 \\
$\Delta \mathrm{SO}^{a}$ & +0.084 & +0.032 & -0.024 & +4.01 \\
$\mathrm{I}-\mathrm{I} \cdots \mathrm{NH}_{3}$ & 2.729 & 2.740 & +0.053 & -8.02 \\
$\Delta \mathrm{SO}^{a}$ & +0.008 & +0.015 & -0.003 & +0.20 \\
$\mathrm{At}-\mathrm{I} \cdots \mathrm{NH}_{3}$ & 2.868 & 2.865 & +0.029 & -4.86 \\
$\Delta \mathrm{SO}^{a}$ & +0.099 & +0.058 & -0.019 & +2.13 \\
$\mathrm{Br}-\mathrm{Br} \cdots \mathrm{NH}_{3}$ & 2.542 & 2.353 & +0.062 & -6.98 \\
$\Delta \mathrm{SO}^{a}$ & +0.000 & +0.003 & -0.000 & +0.00 \\
$\mathrm{At}-\mathrm{Br} \cdots \mathrm{NH}_{3}$ & 2.899 & 2.650 & +0.015 & -2.20 \\
$\Delta \mathrm{SO}^{a}$ & +0.163 & +0.049 & -0.021 & +2.13
\end{tabular}

${ }^{a}$ The spin-orbit coupling effect $(\Delta \mathrm{SO})$ is defined as the difference between the results of $2 \mathrm{c}$ - and sr-calculations.

inclusion of SOC. More precisely, the SOC effects are negligible on the properties of the $\mathrm{Br}_{2} \cdots \mathrm{NH}_{3}$ complex, are small in the case of $\mathrm{I}_{2} \cdots \mathrm{NH}_{3}$, and become sizeable for $\mathrm{At}_{2} \cdots \mathrm{NH}_{3}$ and the other $\mathrm{XY} \cdots \mathrm{NH}_{3}$ complexes. A closer examination reveals that $A t_{2}$ is finally a weaker $\mathrm{XB}$ donor than $\mathrm{I}_{2}\left(\Delta \mathrm{E}^{\mathrm{CP}}=-7.53 \mathrm{kcal} / \mathrm{mol} v \mathrm{~s} .-8.02\right.$ $\mathrm{kcal} / \mathrm{mol}$, respectively), which was confirmed by additional twocomponent relativistic DFT and $a b$ initio calculations (Table S1 in the ESI + ). This behaviour disagrees with both the findings presented above at the scalar-relativistic level of theory, and the assumption that a more polarizable halogen atom would yield to stronger XB interactions. 3,17,40 The magnitude of SOC in astatine is prominent enough to move the lines and it is worth investigating systematically its influence on At-mediated XBs. Thereby, we find that the $\mathrm{At}_{2} \cdots \mathrm{NH}_{3}$ complex is markedly more weakened $(\Delta \mathrm{SO}=+2.15 \mathrm{kcal} / \mathrm{mol})$ than the $\mathrm{I}-\mathrm{At} \cdots \mathrm{NH}_{3}$ one $(\Delta \mathrm{SO}$ $=+0.66 \mathrm{kcal} / \mathrm{mol}$ ), but the SOC effect becomes stronger as the difference in electronegativity between the halogen atoms increases, from iodine to fluorine. For the strongest complex, i.e. $\mathrm{F}-\mathrm{At} \cdots \mathrm{NH}_{3}$, the $\Delta \mathrm{SO}$ contribution to the interaction energy reaches +4.01 $\mathrm{kcal} / \mathrm{mol}$. However, the SOC effect is proportionally more pronounced in the case of the weakest complex: the $\mathrm{XB}$ interaction energy in $\mathrm{At}-\mathrm{Br} \cdots \mathrm{NH}_{3}$ is halved and the $X B$ distance is lengthened by $6.0 \%$. The second most proportionally affected $\mathrm{XB}$ corresponds to the second weakest complex, At $-\cdots \cdots \mathrm{NH}_{3}$, for which the interaction energy is attenuated by one third and the XB distance is lengthened by $3.6 \%$.

Hence, the results from Table 2 and previous works, ${ }^{23,38,39}$ demonstrate the need to take into account the spin-orbit interaction in the quantum mechanical calculations in order to get an accurate description of XBs involving astatinated compounds (this element playing the XB donor role or not). It is noticeable that, among the studied $X B$ complexes, the 
interactions in $\mathrm{At}-\mathrm{Br} \cdots \mathrm{NH}_{3}$ and $\mathrm{At}-\mathrm{I} \cdots \mathrm{NH}_{3}$ are more affected by SOC than in $\mathrm{Br}-\mathrm{At} \cdots \mathrm{NH}_{3}$ and $\mathrm{I}-\mathrm{At} \cdots \mathrm{NH}_{3}$, respectively. More effective relativistic effects are expected for the heaviest halogen element, namely astatine, than for its lighter analogues, iodine and bromine. As a consequence, with Atl and $A t B r$ as $X B$ donors, the SOC would rather be anticipated to have a more important influence on the XBs involving astatine than those mediated by bromine or iodine. Obviously this is not the case, and complementary relativistic calculations confirm for instance that the $\mathrm{XB}$ interaction in $\mathrm{At}-\mathrm{I} \cdots \mathrm{NH}_{3}$ is more weakened by SOC than in I-At $\cdots \mathrm{NH}_{3}$ (Table S1). Such unexpected behaviour, as well as the fact that $A t_{2}$ yields a weaker $\mathrm{XB}$ interaction with ammonia than $I_{2}$ does, can be traced back to the intrinsic properties of the $X Y$ monomers.

\subsection{Rationales from the $X B$ donor's properties}

According to the definition of the International Union of Pure and Applied Chemistry (IUPAC), "a halogen bond occurs when there is evidence of a net attractive interaction between an electrophilic region associated with a halogen atom in a molecular entity and a nucleophilic region in another, or the same, molecular entity". 65 From the seminal studies of Politzer and co-workers, $8,66,67$ investigating the electrophilic region on the outer side of the halogen atom, emerged the fruitful concept of $\sigma$-hole: a region of depleted electron-density and often positive molecular electrostatic potential (MEP). This space region is thus prone to interact with electron-rich sites. The local maximum value at the molecular surface of the MEP $V_{S, \max }$, is a widely used descriptor to characterize the donating ability of XB donors. ${ }^{17,3,68,69,19}$ For instance, the Atl monomer displays a positive region of the MEP concentrated along the AtI axis, both on the astatine and iodine sides (Fig. 1a). The corresponding $V_{\mathrm{s} \text {, max }}$ values are reported in Table 3 , together with those of the other $X Y$ monomers. The $V_{S, \max }$ value at the iodine $\sigma$-hole in Atl (2.62 a.u.) is markedly smaller than the one in $I_{2}(4.78$ a.u.), which is in line with an interaction energy in

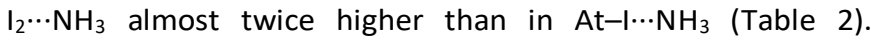
Regarding the astatine $\sigma$-hole, the $V_{s, \max }$ value in Atl (6.82 a.u.) is about half larger than in $A_{2}$ (4.65 a.u.), and similarly the interaction energy is about $40 \%$ stronger in I-At $\cdots \mathrm{NH}_{3}$ than in $\mathrm{At}_{2} \cdots \mathrm{NH}_{3}$. It is also worth noting that, at least for the homonuclear dihalogen systems, the assumption that the $\mathrm{XB}$
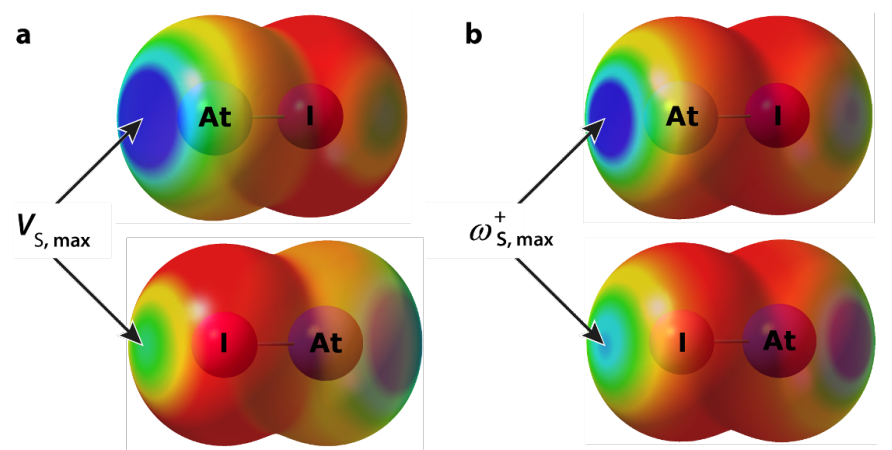

Fig. 1 2c-PW6B95/AVTZ calculated electrostatic potential (a) and local electrophilicity (b) at the Atl molecular surface (defined by 0.001 a.u. isovalues of the electron density). Colour code: from red (lowest values) to blue (highest values).
Table 3 Maximum value of the electrostatic potential $\left(V_{s, \max }\right)$ and of the local electrophilicity $\left(\omega_{S, \max }^{+}\right)$of each XY monomer at the $\sigma$-hole, calculated at the $2 \mathrm{c}$ PW6B95/AVTZ level of theory on the molecular surface defined by 0.001 a.u. isovalue of the electron density

\begin{tabular}{lcccc}
\hline \multicolumn{1}{c}{$\mathrm{XY}$} & $V_{\mathrm{s}, \max }(\mathrm{At})$ & $\boldsymbol{\omega}_{\mathrm{S}, \max }^{+}(\mathrm{At})$ & $V_{\mathrm{s} \text { max }}(\mathrm{Y})$ & $\omega_{\mathrm{s}, \max }^{+}(\mathrm{Y})$ \\
species & $\left(10^{-2}\right.$ a.u. $)$ & $\left(10^{-4}\right.$ a.u. $)$ & $\left(10^{-2}\right.$ a.u. $)$ & $\left(10^{-4} \mathrm{a} . \mathrm{u}.\right)$ \\
\hline At-At & 4.65 & 1.27 & 4.65 & 1.27 \\
$\mathrm{At}-\mathrm{I}$ & 6.82 & 1.68 & 2.62 & 1.06 \\
$\mathrm{At}-\mathrm{Br}$ & 7.97 & 1.98 & 0.99 & 0.92 \\
$\mathrm{At}-\mathrm{Cl}$ & 8.68 & 2.18 & $<0$ & 0.73 \\
$\mathrm{At}-\mathrm{F}$ & 10.20 & 2.65 & $<0$ & 0.44 \\
$\mathrm{I}-\mathrm{I}$ & - & - & 4.78 & 1.46 \\
$\mathrm{Br}-\mathrm{Br}$ & - & - & 4.68 & 1.70 \\
\hline
\end{tabular}

strength increases monotonously with the polarizability of the $\mathrm{XB}$ donor atom ${ }^{38}$ is also contradicted by the analysis of the $V_{\mathrm{s}, \max }$ descriptor. Indeed, the $V_{s, \max }$ values are smaller in $A t_{2}$ than in $I_{2}$ when SOC is accounted for in the calculations, in good agreement with the weaker interaction energy computed for $\mathrm{At}_{2} \cdots \mathrm{NH}_{3}$ vs. $\mathrm{I}_{2} \cdots \mathrm{NH}_{3}$.

However, the characterization of $\mathrm{XB}$ interactions remains misleading and incomplete when only charge-controlled descriptors are used. The magnitude of the charge-transfer component in XB interactions is the subject of an ongoing debate, ${ }^{3,17-19}$ but numerous works have demonstrated by various state-of-the-art analysis schemes its undisputable role, 10,11,13,70-72 which becomes especially significant when the heaviest halogen elements are involved. ${ }^{12,15,40,73}$ In addition to descriptors used to probe the electrostatic contribution, i.e. the charge-control, complementary tools are required to describe the charge-transfer component, i.e. the orbital- or covalentcontrol. ${ }^{74}$ Conceptual density functional theory provided appealing and general-purpose models for chemical reactivity. Following the efforts of Pinter et al. ${ }^{75}$ and Tognetti et al. ${ }^{76}$ to derive, from the Fukui functions, relevant reactivity indices in the context of halogen bonding, we recently proposed to use the local electrophilicity $\omega^{+}(r)$ to characterize the $\sigma$-hole properties. ${ }^{38}$ This function is defined as the product between the global electrophilicity index $\omega\left(\chi^{2} / 2 \eta\right)$ of the system and the $f^{+}(r)$ Fukui function, which shows the distribution of an infinitesimal charge added to the system. ${ }^{58}$ Therefore, a high value of $\omega^{+}(r)$ indicates an electrophilic zone. For instance, the computed values of the local electrophilicity at the molecular surface of Atl clearly highlight the structure of the $\sigma$-holes at both the iodine and astatine sites (Fig. 1b). The local maximum value, $\omega_{S, \max }^{+}$, can be viewed as the complementary analogue of the $V_{S \text {, max }}$ descriptor, dedicated to probe the orbital-control in the XB interaction. ${ }^{38}$ The computed values of $\omega_{S, \text { max }}^{+}$for each XB donor site of the $X Y$ monomers are reported in Table 3 . The relationship between the $X B$ interaction energy in the $X-Y \cdots N_{3}$ complexes and the $\omega_{S, \max }^{+}$value at the $Y$ atom $\sigma$-hole in the $X Y$ monomer is illustrated in Fig. 2. There is an obvious trend showing that the $\mathrm{XB}$ interaction strength increases with $\omega_{\mathrm{s}, \max }^{+}$ for the whole series of $X-Y \cdots N_{3}$ systems (a higher electronegativity of the $X$ atom implying a stronger electrophilicity of the $Y$ site). Nevertheless, with a more detailed inspection of these data, family-dependent relationships clearly appear when the different XB donating sites ( $\mathrm{At}$, I or $\mathrm{Br}$ ) are 


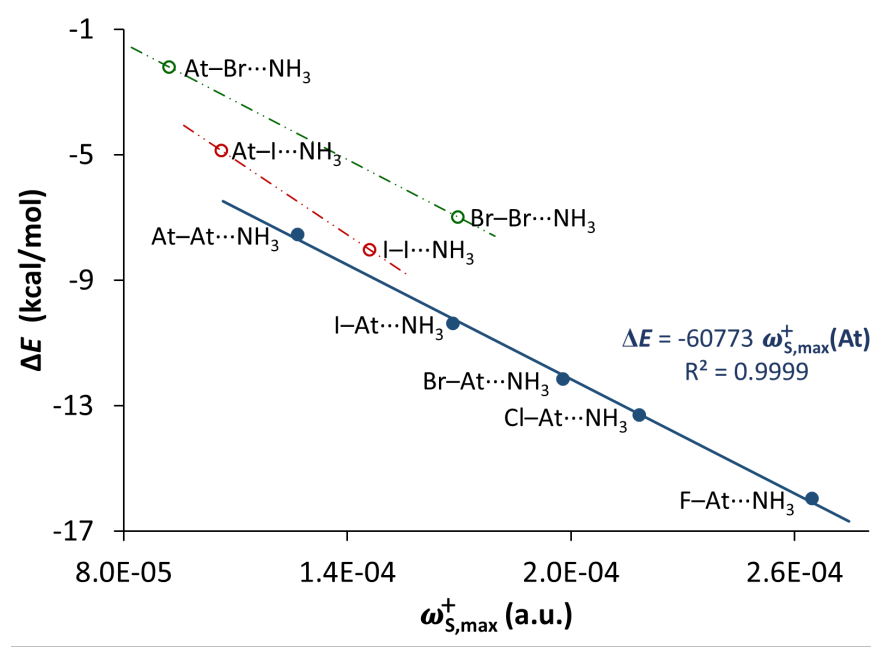

Fig. 2 Correlation between the interaction energy in the $\mathrm{X}-\mathrm{Y} \cdots \mathrm{NH}_{3}$ complexes and the $\omega_{\mathrm{S}, \max }^{+}$value at the $\mathrm{Y}$ atom in $\mathrm{XY}$ monomers (the $\mathrm{XB}$ interaction is mediated by the $Y$ halogen element) computed at the 2c-PW6B95/AVTZ level of theory.

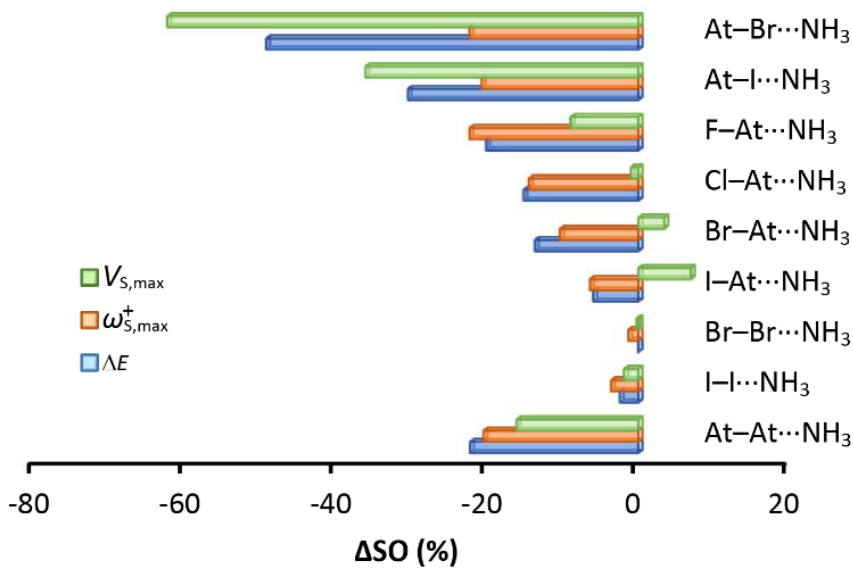

Fig. 3 Influence of the spin-orbit interaction on the interaction energy in the $X-$ $\mathrm{Y} \cdots \mathrm{NH}_{3}$ complexes and on the $\omega_{S, \text { max }}^{+}$and $V_{\mathrm{S}, \max }$ values at the $\mathrm{Y}$ atom in $\mathrm{XY}$ monomers (the $\mathrm{XB}$ interaction is mediated by the $\mathrm{Y}$ halogen element).

distinguished. The interaction energy of the five At-mediated XB complexes is perfectly correlated to the local electrophilicity value at the astatine $\sigma$-hole. Although the iodinated and brominated families only involve two representatives, their data points in Fig. 2 are clearly found above the astatinated family. As a consequence, $\omega_{S, \max }^{+}$appears as a useful descriptor of $\mathrm{XB}$ properties, provided the $\mathrm{XB}$ donor site is kept constant. Note that this family dependence is less pronounced with the electrostatic potential descriptor, $V_{s, \max }$ and the overall correlation is of the same quality than with $\omega_{S, \max }^{+}$(Fig. S1 in the $\mathrm{ESI}+$ ).

We have shown above that SOC markedly affects $X B$ interactions for At-containing compounds, and that it can result in unexpected behaviours. This can also be rationalized on the basis of the chosen descriptors, $\omega_{s, \max }^{+}$and $V_{s, \max }$, whose variations with SOC can be significantly different (Fig. 3). At first, the strong weakening of the interaction energy in $\mathrm{At}_{2} \cdots \mathrm{NH}_{3}$ ($22.2 \%)$, compared to those in $\mathrm{I}_{2} \cdots \mathrm{NH}_{3}(-2.4 \%)$ and $\mathrm{Br}_{2} \cdots \mathrm{NH}_{3}$ ($0.0 \%)$, is corroborated by a strong decrease on $\omega_{s, \max }^{+}(-20.4 \%)$ and $V_{S, \max }(-16.1 \%)$ values calculated for $A t_{2}$, compared to the marginal lessening predicted for $\mathrm{I}_{2}\left(-3.6 \%\right.$ on $\omega_{S, \max }^{+}$and $-1.9 \%$ on $\left.V_{s, \max }\right)$ and $\mathrm{Br}_{2}\left(-1.3 \%\right.$ on $\omega_{s, \max }^{+}$and $-0.3 \%$ on $\left.V_{s, \max }\right)$. If there is an overall consistency between the evolutions of the interaction energies and of these two descriptors, the predicted increase of $V_{s, \max }(\mathrm{At})$ in $\mathrm{Atl}$ and $\mathrm{AtBr}$ monomers, when the SOC is taken into account in the calculations, is nevertheless inconsistent with the concomitant weakening of the XBs in IAt $\cdots \mathrm{NH}_{3}$ and $\mathrm{Br}-\mathrm{At} \cdots \mathrm{NH}_{3}$. For these complexes, the chargetransfer contribution to the $\mathrm{XB}$ interaction cannot be ignored anymore. The $\omega_{S, \max }^{+}$descriptor appears to better describe the complexes weakening as its value at the astatine $\sigma$-hole decreases by $6.4 \%$ and $10.4 \%$ in $\mathrm{Atl}$ and $\mathrm{AtBr}$, respectively, corroborating the respective attenuations by $6.0 \%$ and $13.7 \%$ of the $\mathrm{I}-\mathrm{At} \cdots \mathrm{NH}_{3}$ and $\mathrm{Br}-\mathrm{At} \cdots \mathrm{NH}_{3}$ interaction energies, respectively. Furthermore, it is clear from Fig. 3 that the $\omega_{S, \max }^{+}$ descriptor (as well as $V_{S, \max }$ ) is much more affected by SOC at the iodine and bromine atoms in Atl and AtBr, leading to an XB donating ability significantly more sensitive to SOC for the lighter halogen atom than for astatine. These findings support the stronger weakening of the interaction energy predicted for At $-\mathrm{\cdots} \cdots \mathrm{NH}_{3}$ and $\mathrm{At}-\mathrm{Br} \cdots \mathrm{NH}_{3}$, as well as the larger lengthening of the $\mathrm{XB}$ distances (Table 2), with respect to the I-At $\cdots \mathrm{NH}_{3}$ and $\mathrm{Br}-\mathrm{At} \cdot \cdots \mathrm{NH}_{3}$ complexes. Therefore, the intrinsic characteristics of the $\sigma$-hole and their study for the XY monomers allow us to anticipate the properties of the complexes involving these $\mathrm{XB}$ donors.

\subsection{Relationship with charge-shift bonding}

This section is intended to shed light on bonding mechanisms occurring in astatinated compounds and on their influence on the ability of these compounds to interact through XBs. We first focus on the $A t_{2}$ species, which was previously shown to belong to the charge-shift bonding systems. ${ }^{29,77}$ Charge-shift (CS) bonds constitute a class of bond different from the covalent and ionic ones, where the bond stability is achieved by large and dynamic fluctuations of the bonding electron-pair, i.e. a large resonance between the covalent and ionic Lewis structures $\left(-X \mid Y^{+} \leftrightarrow X-\right.$ $\left.Y \leftrightarrow{ }^{+} X \mid Y^{-}\right)$. CS bonding has been introduced initially by Shaik et al. in the framework of valence bond theory, ${ }^{78}$ and typical signatures have later been found in the context of the QTAIM and ELF topological approaches. ${ }^{79-81}$

The ELF localization domains determined for $A t_{2}$ are displayed in Fig. 4. The topology is typical of most diatomics with two core basins, two non-bonding valence basins and one bonding basin. The electron populations of the ELF basins, presented in Table 4, are in very close agreement with previous results obtained at the 2c-B3LYP/AVTZ level of theory. ${ }^{29} \mathrm{~A}$ similar analysis can therefore be conducted, which reveals

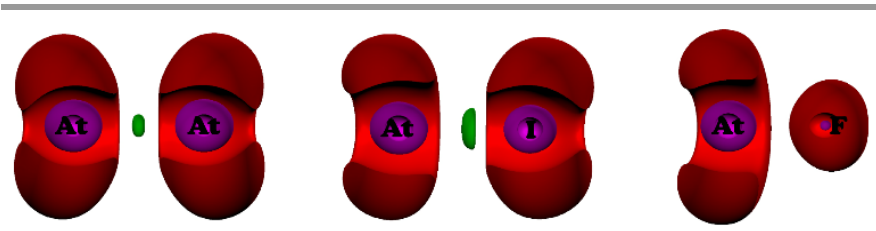

Fig. 4 Split of ELF localization domains (isosurface $=0.55$ ) of the At $Y$ species $(Y=A t$, I, F) calculated at the 2c-PW6B95/AVTZ level of theory. Color code: magenta for core $\mathrm{C}(\mathrm{Y})$ basins, red for valence $\mathrm{V}(\mathrm{Y})$ basins and green for bonding $\mathrm{V}(\mathrm{At}, \mathrm{Y})$ basins. 
Table 4 ELF electron population analysis of AtY species $(\mathrm{Y}=\mathrm{At}, \mathrm{I}, \mathrm{Br}, \mathrm{Cl}, \mathrm{F})$ obtained at the 2c-PW6B95/AVTZ level of theory

\begin{tabular}{cccccc} 
& \multicolumn{3}{c}{ Non-bonding } & & Bonding \\
\cline { 2 - 3 } \cline { 6 - 6 } basins & $\mathrm{C}(\mathrm{At})+\mathrm{V}(\mathrm{At})$ & $\mathrm{C}(\mathrm{Y})+\mathrm{V}(\mathrm{Y})$ & $\mathrm{pop} \pi^{b}$ & & $\mathrm{~V}(\mathrm{At}, \mathrm{Y})$ \\
\hline $\mathrm{At}_{2}$ & 24.71 & 24.71 & 7.90 & & 0.58 \\
$\Delta \mathrm{SO}^{a}$ & +0.18 & +0.18 & +1.70 & & -0.36 \\
$\mathrm{Atl}$ & 24.48 & 24.80 & 7.09 & & 0.72 \\
$\Delta \mathrm{SO}^{a}$ & +0.03 & +0.23 & +1.48 & & -0.26 \\
$\mathrm{AtBr}$ & 24.42 & 25.04 & 7.53 & & 0.54 \\
$\Delta \mathrm{SO}^{a}$ & +0.02 & +0.30 & +0.70 & & -0.32 \\
$\mathrm{AtCl}$ & 24.38 & 17.27 & 6.46 & & 0.35 \\
$\Delta \mathrm{SO}^{a}$ & +0.03 & +0.33 & +0.50 & & -0.36 \\
$\mathrm{AtF}$ & 24.33 & 9.67 & 7.00 & & $c$ \\
$\Delta \mathrm{SO}^{a}$ & -0.01 & +0.01 & +0.36 &
\end{tabular}

${ }^{a}$ The spin-orbit effects are defined as the difference between the results of 2c- and sr-calculations. ${ }^{b}$ The $\pi$ population of the valence basins $\mathrm{V}(\mathrm{At})+\mathrm{V}(\mathrm{Y})$ is evaluated by only taking into account the expansion coefficients of the $p_{x}$ and $p_{y}$ gaussian basis functions during the integration of the electron density over the basin volumes. ${ }^{c}$ No ELF basin of this type was found.

notably some features of CS bonding. The population of the $\mathrm{V}(\mathrm{At}, \mathrm{At})$ bonding basin, calculated by integrating the electron density over the basin volume, is for example particularly depleted $(0.58 \mathrm{e})$ and deviates significantly from the 2 electrons expected for a classical covalent single bond. It is also worth noting that the $\mathrm{V}(\mathrm{At}, \mathrm{At})$ basin population dramatically falls, by $\sim 40 \%$, as a result of the spin-orbit interaction, consequently fading the covalent component to the bond. The resulting electronic redistribution is in favour of the $\pi$ population $(+1.70$ e) within the non-bonding $\mathrm{V}(\mathrm{At})$ basins: we previously depicted it as an electron withdrawal from the covalent $\sigma$-bond (-0.36 e), and the $\sigma$ lone-pairs $(-1.34 \mathrm{e})$, to the valence $\pi$ system located in the lone-pair regions. ${ }^{29,36,77}$ In fact, such mechanism closely follows the lone-pair bond-weakening effect (LPBWE), 82 which results from Pauli repulsion between the bonding electrons and the $\sigma$ lone-pairs adjacent to the bond. This repulsion increases the bond kinetic energy beyond the virial ratio. The stabilization is finally achieved by enhancing the ionic-covalent mixing, which gives rise to CS bonding. ${ }^{78,81}$ Hence, SOC increases the CS character of the bond in $A t_{2}$, while weakening the ability of astatine to participate in covalent interactions. The LPBWE is expected to hinder any electron transfer from a nucleophilic reactant to the $\sigma$ system in $A t_{2}$, resulting in a local electrophilicity lessening at the astatine $\sigma$-hole. As discussed above, the $\omega_{S, \max }^{+}$value is indeed strongly decreased when SOC is included in the calculations. QTAIM descriptors calculated for the $\mathrm{At}_{2} \cdots \mathrm{NH}_{3}$ complex (Table 5) confirm the decrease of charge transfer from $\mathrm{NH}_{3}$ to the $\mathrm{At}_{2}$ fragment, i.e. the weakening with SOC of the covalent component to the XB interaction. The $A t_{2}$ fragment bears a slightly less negative charge $(\Delta S O=+0.01 \mathrm{e})$. The $X \mathrm{~B}$ interaction is characterized by a so-called "bond path" 61,83 between the At and $\mathrm{N}$ atoms and the electron density at the corresponding bond critical point (BCP, the point of minimum electron density on the bond path) is decreased by SOC by $\sim 18 \%$. The $\Delta E$ interaction energy in $\mathrm{At}_{2} \cdots \mathrm{NH}_{3}$ is consequently strongly weakened (by $-22.2 \%$ as discussed
Table 5 Selected QTAIM descriptors obtained at the 2c-PW6B95/AVTZ level of theory for $\mathrm{XY} \cdots \mathrm{NH}_{3}$ complexes $(\mathrm{X}, \mathrm{Y}=\mathrm{At}, \mathrm{I})$

\begin{tabular}{|c|c|c|c|}
\hline & \multirow{2}{*}{$\begin{array}{c}\text { Integrated } \\
\text { property } \\
\mathrm{q}(\mathrm{XY})^{b}\end{array}$} & \multicolumn{2}{|c|}{$\begin{array}{c}\text { At the } \mathrm{BCP} \text { of the } \mathrm{Y} \cdots \mathrm{N} \\
\text { interaction }\end{array}$} \\
\hline & & $\rho_{\mathrm{b}} \cdot 10^{2 c}$ & $\left|V_{\mathrm{b}}\right| / G_{\mathrm{b}}{ }^{d}$ \\
\hline $\mathrm{At}-\mathrm{At} \cdots \mathrm{NH}_{3}$ & -0.06 & 2.7 & 1.02 \\
\hline$\Delta \mathrm{SO}^{a}$ & +0.01 & -0.6 & -0.05 \\
\hline $\mathrm{I}-\mathrm{At} \cdots \mathrm{NH}_{3}$ & -0.07 & 3.2 & 1.05 \\
\hline$\Delta \mathrm{SO}^{a}$ & +0.02 & -0.3 & -0.04 \\
\hline At $-1 \cdots \mathrm{NH}_{3}$ & -0.07 & 2.5 & 0.99 \\
\hline$\Delta \mathrm{SO}^{a}$ & +0.02 & -0.5 & -0.06 \\
\hline \multicolumn{4}{|c|}{$\begin{array}{l}{ }^{a} \text { The spin-orbit effects are defined as the difference between the results of } 2 \mathrm{c} \text { - and } \\
\text { sr-calculations. }{ }^{b} \text { Sum of } X \text { and } Y \text { atomic charges (a.u.). }{ }^{c} \text { Electron density (a.u.). }{ }^{d} \\
\text { Ratio between the potential energy density }\left(V_{b}\right) \text { and the positive definite kinetic } \\
\text { energy density }(G \text { b). }\end{array}$} \\
\hline
\end{tabular}

previously). We can therefore conclude that the CS bonding, markedly enhanced by $\mathrm{SOC}$ in $A t_{2}$, is responsible for the lower $X B$ donating ability of $A t_{2}$ with respect to $I_{2}$.

Within the heteronuclear dihalogen series, the CS bonding was also shown to play a crucial role in the case of the Atl species. ${ }^{29}$ The ELF topology of Atl is similar to that of $A t_{2}$ (Fig. 4), with especially $(\mathrm{i})$ a depleted $\mathrm{V}(\mathrm{At}, \mathrm{l})$ bonding basin $(0.72 \mathrm{e}$, Table 4), and (ii) a spectacular electron withdrawal from this basin (0.26 e) to the valence $\pi$ system related to the $V(A t)$ and $V(I)$ nonbonding basins (the $\pi$ population increases by +1.48 e) as a result of SOC. This electronic redistribution is clearly in line with the LPBWE. Therefore, the spin-orbit interaction weakens again the covalent component and enhances the CS bonding, which ultimately lowers the XB donating ability of Atl. However, it is not yet rationalized why the $\sigma$-hole at the iodine atom appears more affected. As previously shown, ${ }^{33}$ the SOC decreases astatine electronegativity by $\sim 10 \%$, and from the QTAIM analysis of the Atl species (Table S2 in the ESI + ), the astatine charge $q(A t)$ increases from +0.09 e to +0.19 e. The electronic redistribution induced by the spin-orbit interaction is therefore essentially directed towards the iodine atom and the $\mathrm{V}(\mathrm{I})$ population is, for instance, increased by $+0.20 \mathrm{e}$. In other words, because the difference of electronegativity between the two atoms in Atl is significantly increased, most of the consequences of the spin-orbit interaction (mainly due to astatine coreelectrons) appear to be transmitted to the iodine atom. Then, the local electrophilicity $\omega_{s, \max }^{+}$at the iodine $\sigma$-hole is more decreased, justifying the larger weakening of the $\mathrm{XB}$ interaction

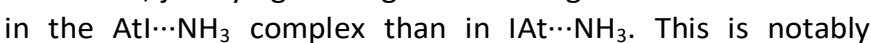
corroborated by QTAIM descriptors related to the charge transfer in both complexes (Table 5). For instance, the ratio between the potential energy density $(V)$ and the (positive definite) kinetic energy density $(G)$ at the BCP, $\left|V_{\mathrm{b}}\right| / G_{\mathrm{b}}$, reflects the covalency magnitude of the interaction:83,84 $\left|V_{b}\right| / G_{b}>1$ indicates that the potential energy density is the leading term and electrons are stabilized at the $\mathrm{BCP}$. Regarding the $\mathrm{XB}$ interactions in $\mathrm{AtI} \cdots \mathrm{NH}_{3}$ and IAt $\cdots \mathrm{NH}_{3}$, this ratio decreases when SOC is taken into account and it even becomes slightly smaller than 1 for the I-mediated XB, eventually indicating in this case no-covalency. The same analysis carried out for $\mathrm{AtBr}$ and its 
related XB complexes with ammonia leads to similar conclusions.

The situation is less clear cut for $\mathrm{AtCl}$, which also exhibits similarities with AtF: no stable $\mathrm{Cl}$-mediated XB with ammonia exists at the 2c-relativistic level, and the ionic character of the bond becomes strong as witnessed by the significant astatine partial charge $(\mathrm{q}(\mathrm{At})=+0.43$ e, Table S2). Regarding the AtF species, the current QTAIM and ELF analyses (Tables 4 and S2) agree for a bond previously characterized as mainly ionic. ${ }^{29}$ Note that this bond seems to be the least affected by SOC according to lesser changes on ELF and QTAIM descriptors (see also the influence on the spectroscopic constants, Table S3 in the ESI + ), but the LPBWE is still exalted by the spin-orbit interaction (noticeable increase of the $V(A t)$ and $V(F) \pi$ populations). The effects become analogous on the astatine and fluorine $\sigma$-holes $\left(\omega_{s, \max }^{+}\right.$lowered by $-22.3 \%$ and $-19.1 \%$, respectively)

\section{Conclusions}

By means of two-component relativistic quantum mechanical calculations, we have scrutinized the influence of the main spindependent relativistic effect on halogen-bond (XB) interactions formed between a series of At-containing dihalogens ( $X Y$, with $\mathrm{X}, \mathrm{Y}=\mathrm{At}, \mathrm{I}, \mathrm{Br}, \mathrm{Cl}$ and $\mathrm{F}$ ) and ammonia. The spin-orbit coupling (SOC) was found to systematically weaken the strength of the studied XBs. For instance, the interaction energy is reduced by more than $20 \%$ in the $A t_{2} \cdots \mathrm{NH}_{3}$ complex, and $A t_{2}$ is finally a weaker XB donor than its lighter analogue, $I_{2}$. SOC effects are even stronger for the heteronuclear dihalogens, and, astonishingly, the $\mathrm{XBs}$ in the $\mathrm{At}-\mathrm{Br} \cdots \mathrm{NH}_{3}$ and $\mathrm{At}-\mathrm{I} \cdots \mathrm{NH}_{3}$ complexes are more affected than in $\mathrm{Br}-\mathrm{At} \cdots \mathrm{NH}_{3}$ and I-At $\cdots \mathrm{NH}_{3}$, respectively. We have shown that some rationales emerge from intrinsic descriptors of the $\mathrm{XB}$ donors, calculated at the so-called " $\sigma$-hole" of the atoms mediating the XB interactions. If the magnitude of the $V_{\mathrm{s} \text {, max }}$ electrostatic descriptor, commonly used for $\mathrm{XB}$ interactions characterization, does not forecast the more important SOC influence on the XBs mediated by bromine in $\mathrm{AtBr}$ and iodine in $\mathrm{Atl}$, all observed trends are well mirrored by a recently introduced local electrophilicity index ${ }^{38} \omega_{\mathrm{s}, \max }^{+}$. This outcome highlights the role of the charge-transfer component in the XB interactions mediated by At-compounds.

Including the spin-orbit interaction in the calculations allows us to uncover the importance of the bonding mechanism in $X Y$ on the $X B$ properties of these donors, through the analysis of $S O C$ effects on chemical bond descriptors arising from the quantum chemical topology. Our results show in $\mathrm{At}_{2}$ and AtI species that SOC enhances the charge-shift (CS) character of the bond, i.e. it enhances the Pauli repulsion between the bonding $\sigma$-electrons and the adjacent lone-pairs that have the same symmetry. The resulting effect is an electron withdrawal from the covalent $\sigma$-bond, and the $\sigma$ lone-pairs, to the valence $\pi$ system located in the lone-pair regions. Hence, the CS mechanism strengthened by the spin-orbit interaction (i) weakens locally the electrophilicity at the $\sigma$-hole of the halogen atom (i.e. $\omega_{s, \max }^{+}$), and consequently, (ii) hinders electron transfer from a Lewis bases, such as $\mathrm{NH}_{3}$, to the $\mathrm{XB}$ donor. It comes out that the CS bonding is responsible for the lower $\mathrm{XB}$ donating ability of $A t_{2}$ with respect to $I_{2}$. For the Atl heteronuclear dihalogen, the SOC significantly increases the electronegativity difference between astatine and iodine atoms. The above-mentioned electronic redistribution, induced by the SOC, then occurs predominantly towards iodine. Therefore, the $\omega_{s, \max }^{+}$value at the iodine $\sigma$-hole is more decreased than at the astatine $\sigma$-hole, and the At- $-\cdots \mathrm{NH}_{3} \mathrm{XB}$ complex is consequently more affected than the I-At $\cdots \mathrm{NH}_{3}$ one.

A fundamental question arising from these observations is whether this connection between $\mathrm{CS}$ bonding and $\mathrm{XB}$ interactions is closely related to heavy halogens or more general. Indeed, fluorine is the lightest halogen element and molecular fluorine is an archetypal CS bonding system. ${ }^{78-80} F_{2}$ is also known to be, at the best, a very weak XB donor. ${ }^{13,14,16,40}$ is the CS bonding mechanism the cause of the poor capability of fluorine to engage in XBs? This hypothesis is currently under active investigations.

\section{Conflicts of interest}

There are no conflicts to declare.

\section{Acknowledgements}

This work has been supported in part by grants from the French National Agency for Research called "Programme d'Investissements d'Avenir" (ANR-11-EQPX-0004, ANR-11LABX-0018). It was carried out using HPC resources from GENClCINES/IDRIS (grant A0020805117) and from CCIPL ("Centre de Calcul Intensif des Pays de la Loire").

\section{References}

1 T. M. Beale, M. G. Chudzinski, M. G. Sarwar and M. S. Taylor, Chem. Soc. Rev., 2013, 42, 1667-1680.

2 R. Wilcken, M. O. Zimmermann, A. Lange, A. C. Joerger and F. M. Boeckler, J. Med. Chem., 2013, 56, 1363-1388.

3 G. Cavallo, P. Metrangolo, R. Milani, T. Pilati, A. Priimagi, G. Resnati and G. Terraneo, Chem. Rev., 2016, 116, 2478-2601.

4 B. Li, S.-Q. Zang, L.-Y. Wang and T. C. W. Mak, Coord. Chem. Rev., 2016, 308, 1-21.

5 D. Bulfield and S. M. Huber, Chem. Eur. J., 2016, 22, 1443414450.

6 K. T. Mahmudov, M. N. Kopylovich, M. F. C. Guedes da Silva and A. J. L. Pombeiro, Coord. Chem. Rev., 2017, 345, 54-72.

7 E. Persch, O. Dumele and F. Diederich, Angew. Chem., Int. Ed., 2015, 54, 3290-3327.

8 T. Clark, M. Hennemann, J. S. Murray and P. Politzer, J. Mol. Model., 2007, 13, 291-296.

9 H. Torii and M. Yoshida, J. Comput. Chem., 2010, 31, 107-116.

10 L. P. Wolters and F. M. Bickelhaupt, ChemistryOpen, 2012, 1, 96105.

11 O. A. Syzgantseva, V. Tognetti and L. Joubert, J. Phys. Chem. A, 2013, 117, 8969-8980.

12 M. H. Kolár̆, P. Deepa, H. Ajani, A. Pecina and P. Hobza, in Halogen Bonding II, Springer Cham, 2014, vol. 359, pp. 1-25.

13 C. Wang, D. Danovich, Y. Mo and S. Shaik, J. Chem. Theory Comput., 2014, 10, 3726-3737.

14 K. Eskandari and M. Lesani, Chem. Eur. J., 2015, 21, 4739-4746. 
15 J. Řezáč and A. de la Lande, Phys. Chem. Chem. Phys., 2016, 19, 791-803.

16 V. Oliveira, E. Kraka and D. Cremer, Phys. Chem. Chem. Phys., 2016, 18, 33031-33046.

17 P. Politzer, J. S. Murray and T. Clark, Phys. Chem. Chem. Phys., 2010, 12, 7748-7757.

18 L. P. Wolters, P. Schyman, M. J. Pavan, W. L. Jorgensen, F. M. Bickelhaupt and S. Kozuch, Wiley Interdiscip. Rev. Comput. Mol. Sci., 2014, 4, 523-540.

19 M. H. Kolář and P. Hobza, Chem. Rev., 2016, 116, 5155-5187.

20 L. Öhrström and J. Reedijk, Pure Appl. Chem., 2016, 88, 12251229.

21 D. S. Wilbur, Nat. Chem., 2013, 5, 246.

22 G. Vaidyanathan and M. R. Zalutsky, Curr. Radiopharm., 2008, 1, 177-196.

23 N. Guo, R. Maurice, D. Teze, J. Graton, J. Champion, G. Montavon and N. Galland, Nat. Chem., 2018, 10, 428-434.

24 T. Fleig and A. J. Sadlej, Phys. Rev. A, 2002, 65, 032506.

25 T. Ayed, M. Seydou, F. Réal, G. Montavon and N. Galland, J. Phys. Chem. B, 2013, 117, 5206-5211.

26 A. Hermann, R. Hoffmann and N. W. Ashcroft, Phys. Rev. Lett. 2013, 111, 116404.

27 T. Ayed, F. Réal, G. Montavon and N. Galland, J. Phys. Chem. B, 2013, 117, 10589-10595.

28 A. Severo Pereira Gomes, F. Réal, N. Galland, C. Angeli, R. Cimiraglia and V. Vallet, Phys. Chem. Chem. Phys., 2014, 16, 9238-9248.

29 J. Pilmé, E. Renault, F. Bassal, M. Amaouch, G. Montavon and N. Galland, J. Chem. Theory Comput., 2014, 10, 4830-4841.

30 R. Maurice, F. Réal, A. Severo Pereira Gomes, V. Vallet, G. Montavon and N. Galland, J. Chem. Phys., 2015, 142, 094305.

31 D.-C. Sergentu, F. Réal, G. Montavon, N. Galland and R. Maurice, Phys. Chem. Chem. Phys., 2016, 18, 32703-32712.

32 R. Ramírez-Tagle, L. Alvarado-Soto, A. Villavicencio-Wastavino and L. Alvarez-Thon, Phys. Chem. Chem. Phys., 2016, 18, 2575125755.

33 S. Dumitru-Claudiu, D. Grégoire, M. Gilles, M. Rémi and G. Nicolas, J. Comput. Chem., 2016, 37, 1345-1354.

34 J. Champion, M. Seydou, A. Sabatié-Gogova, E. Renault, G. Montavon and N. Galland, Phys. Chem. Chem. Phys., 2011, 13, 14984-14992.

35 P. Norman, B. Schimmelpfennig, K. Ruud, H. J. A. Jensen and H. Ågren, J. Chem. Phys., 2002, 116, 6914-6923.

36 J. Pilmé, E. Renault, T. Ayed, G. Montavon and N. Galland, J. Chem. Theory Comput., 2012, 8, 2985-2990.

37 F. Réal, A. Severo Pereira Gomes, Y. O. Guerrero Martínez, T. Ayed, N. Galland, M. Masella and V. Vallet, J. Chem. Phys., 2016, 144, 124513.

38 N. Galland, G. Montavon, J.-Y. Le Questel and J. Graton, New J. Chem., 2018, 42, 10510-10517.

39 P. Matczak, Mol. Phys., 2018, 116, 338-350.

40 J. G. Hill and X. Hu, Chem. Eur. J., 2013, 19, 3620-3628.

41 W. Liu, Mol. Phys., 2010, 108, 1679-1706.

42 T. Saue, ChemPhysChem, 2011, 12, 3077-3094.

43 M. Dolg and X. Cao, Chem. Rev., 2012, 112, 403-480.

44 Y. J. Choi and Y. S. Lee, J. Chem. Phys., 2003, 119, 2014-2019.

45 A. V. Mitin and C. van Wüllen, J. Chem. Phys., 2006, 124, 064305

46 D. Peng, W. Liu, Y. Xiao and L. Cheng, J. Chem. Phys., 2007, 127, 104106.

47 D.-D. Yang and F. Wang, Phys. Chem. Chem. Phys., 2012, 14, 15816-15825.

48 Y. Zhao and D. G. Truhlar, J. Phys. Chem. A, 2005, 109, 56565667.

49 T. P. Straatsma, E. Apra, T. L. Windus, E. J. Bylaska, W. A. de Jong, S. Hirata, M. Valiev, M. Hackler, L. Pollack and R. J. Harrison, NWChem, A Computational Chemistry Package for Parallel Computers, Pacific Northwest National Laboratory: Richland, Washington, 2008.
50 K. A. Peterson, D. Figgen, E. Goll, H. Stoll and M. Dolg, J. Chem. Phys., 2003, 119, 11113-11123.

51 K. A. Peterson, B. C. Shepler, D. Figgen and H. Stoll, J. Phys. Chem A, 2006, 110, 13877-13883.

52 M. K. Armbruster, W. Klopper and F. Weigend, Phys. Chem. Chem. Phys., 2006, 8, 4862-4865.

53 T. H. Dunning, J. Chem. Phys., 1989, 90, 1007-1023.

54 R. A. Kendall, T. H. Dunning and R. J. Harrison, J. Chem. Phys., 1992, 96, 6796-6806.

55 D. E. Woon and T. H. Dunning, J. Chem. Phys., 1993, 98, 13581371.

56 S. F. Boys and F. Bernardi, Mol. Phys., 1970, 19, 553-566.

57 TURBOMOLE, a development of University of Karlsruhe and Forschungszentrum Karlsruhe $\mathrm{GmbH}, 1989-2007$, TURBOMOLE $\mathrm{GmbH}$, since 2007, available from http://www.turbomole.com, 2014.

58 P. K. Chattaraj, U. Sarkar and D. R. Roy, Chem. Rev., 2006, 106, 2065-2091.

59 A. D. Becke and K. E. Edgecombe, J. Chem. Phys., 1990, 92, 53975403.

60 B. Silvi and A. Savin, Nature, 1994, 371, 683-686.

61 R. F. W. Bader, Atoms in Molecules: A Quantum Theory, Oxford University Press, New York, USA, 1994.

62 R. J. Gillespie and E. A. Robinson, J. Comput. Chem., 2007, 28, 8797.

63 S. Noury, X. Krokidis, F. Fuster and B. Silvi, Comput. Chem., 1999, 23, 597-604.

64 M. Mantina, A. C. Chamberlin, R. Valero, C. J. Cramer and D. G. Truhlar, J. Phys. Chem. A, 2009, 113, 5806-5812.

65 G. R. Desiraju, P. S. Ho, L. Kloo, A. C. Legon, R. Marquardt, P. Metrangolo, P. Politzer, G. Resnati and K. Rissanen, Pure Appl. Chem., 2013, 85, 1711-1713.

66 T. Brinck, J. S. Murray and P. Politzer, Int. J. Quantum Chem., 1992, 44, 57-64.

67 J. S. Murray, K. Paulsen and P. Politzer, Proc. Indian Acad. Sci. (Chem. Sci.), 1994, 106, 267-275.

68 K. E. Riley, J. S. Murray, P. Politzer, M. C. Concha and P. Hobza, J. Chem. Theory Comput., 2009, 5, 155-163.

69 M. G. Sarwar, B. Dragisic, L. J. Salsberg, C. Gouliaras and M. S. Taylor, J. Am. Chem. Soc., 2010, 132, 1646-1653.

70 S. M. Huber, E. Jimenez-Izal, J. M. Ugalde and I. Infante, Chem. Commun., 2012, 48, 7708-7710.

71 S. Kozuch and J. M. L. Martin, J. Chem. Theory Comput., 2013, 9, 1918-1931.

72 V. Tognetti and L. Joubert, Theor. Chem. Acc., 2016, 135, 124.

73 J. Thirman, E. Engelage, S. M. Huber and M. Head-Gordon, Phys. Chem. Chem. Phys., 2018, 20, 905-915.

74 F. Guégan, P. Mignon, V. Tognetti, L. Joubert and C. Morell, Phys. Chem. Chem. Phys., 2014, 16, 15558-15569.

75 B. Pinter, N. Nagels, W. A. Herrebout and F. De Proft, Chem. Eur. J., 2013, 19, 519-530.

76 V. Tognetti, C. Morell and L. Joubert, Journal of Computational Chemistry, 2015, 36, 649-659.

77 M. Amaouch, E. Renault, G. Montavon, N. Galland and J. Pilmé, in Applications of Topological Methods in Molecular Chemistry, Springer, Cham, 2016, vol. 22, pp. 553-582.

78 S. Shaik, D. Danovich, W. Wu and P. C. Hiberty, Nat. Chem., 2009, 1, 443-449.

79 S. Shaik, D. Danovich, B. Silvi, D. L. Lauvergnat and P. C. Hiberty, Chem. Eur. J., 2005, 11, 6358-6371.

80 L. Zhang, F. Ying, W. Wu, P. C. Hiberty and S. Shaik, Chem. Eur. J., 2009, 15, 2979-2989.

81 S. Shaik, D. Danovich, B. Braida, W. Wu and P. C. Hiberty, in The Chemical Bond II, Springer, Cham, 2015, vol. 170, pp. 169-211.

82 R. T. Sanderson, Polar Covalence, Academic Press, 1983.

83 C. F. Matta and R. J. Boyd, The Quantum Theory of Atoms in Molecules: From Solid State to DNA and Drug Design, Wiley-VCH, Weinheim, 2007. 
84 D. Cremer and E. Kraka, Angew. Chem., Int. Ed., 1984, 23, 627628. 